\title{
Gravitational tests of the generalized uncertainty principle
}

\author{
Fabio Scardigli $^{1,2, a}$, Roberto Casadio ${ }^{3,4, b}$ \\ ${ }^{1}$ Department of Mathematics, College of Engineering, American University of the Middle East, P.O. Box 220, 15453 Dasman, Kuwait \\ ${ }^{2}$ Dipartimento di Matematica, Politecnico di Milano, Piazza Leonardo da Vinci 32, 20133 Milan, Italy \\ ${ }^{3}$ Dipartimento di Fisica e Astronomia, Alma Mater Università di Bologna, via Irnerio 46, 40126 Bologna, Italy \\ ${ }^{4}$ INFN, Sezione di Bologna, viale Bert Pichat 6/2, 40127 Bologna, Italy
}

Received: 18 April 2015 / Accepted: 18 August 2015 / Published online: 15 September 2015

(c) The Author(s) 2015. This article is published with open access at Springerlink.com

\begin{abstract}
We compute the corrections to the Schwarzschild metric necessary to reproduce the Hawking temperature derived from a generalized uncertainty principle (GUP), so that the GUP deformation parameter is directly linked to the deformation of the metric. Using this modified Schwarzschild metric, we compute corrections to the standard general relativistic predictions for the light deflection and perihelion precession, both for planets in the solar system and for binary pulsars. This analysis allows us to set bounds for the GUP deformation parameter from well-known astronomical measurements.
\end{abstract}

\section{Introduction}

Research on generalizations of the uncertainty principle of quantum mechanics has nowadays a long history [1-4]. One of the main lines of investigation focuses on understanding how the Heisenberg uncertainty principle (HUP) should be modified once gravity is taken into account. Given the pivotal rôle played by gravitation in these arguments, it is not surprising that the most relevant modifications to the HUP have been proposed in string theory, loop quantum gravity, deformed special relativity, and studies of black hole physics [5-16], just to mention some of the most notable frameworks.

An interesting novelty, emerged during the last decade or so, is a lively debate on the measurable features of various kinds of generalized uncertainty principles (GUPs). From more theoretical shores, the discussion has therefore landed on the ground of experimental predictions about the size of these modifications, and several experiments have been proposed to test GUPs in the laboratory. Among the more elaborated proposals are those, for example, of the groups of Brukner, Cerdonio, and Bonaldi [17-19].

\footnotetext{
a e-mail: fabio@phys.ntu.edu.tw

be-mail: casadio@bo.infn.it
}

Studies that aim at putting bounds on the dimensionless deforming parameter of the GUP, heretofore denoted by $\beta$, date back at least to Brau [20] and can be roughly divided into three different categories (actually, only two, as we will see). In the first group one finds papers such as those of Brau [20], Vagenas [21,22], Nozari [23], which use a specific (in general, non-linear) representation of the operators in the deformed fundamental commutator ${ }^{1}$

$[\hat{X}, \hat{P}]=i \hbar\left(1+\beta \frac{\hat{P}^{2}}{m_{\mathrm{p}}^{2}}\right)$,

in order to compute corrections to quantum mechanical predictions, such as energy shifts in the spectrum of the hydrogen atom, or to the Lamb shift, the Landau levels, scanning tunneling microscope, charmonium levels, etc. As we shall see in Sect. 5, the bounds so obtained on $\beta$ are quite stringent, but the drawback of this approach is a potentially strong dependence of the expected shifts on the specific (non-linear) representation chosen for the operators $\hat{X}$ and $\hat{P}$ in the fundamental commutator (1).

In the second group, we can find the works of, e.g., Chang [24], Nozari and Pedram [25,26], where a deformation of classical Newtonian mechanics is introduced by modifying the standard Poisson brackets in a way that resembles the quantum commutator,

$[\hat{x}, \hat{p}]=i \hbar\left(1+\beta_{0} \hat{p}^{2}\right) \Rightarrow\{X, P\}=\left(1+\beta_{0} P^{2}\right)$,

where $\beta_{0}=\beta / m_{\mathrm{p}}{ }^{2}$. In particular, Chang in Ref. [24] computes the precession of the perihelion of Mercury directly from this GUP-deformed Newtonian mechanics, and interprets it as an extra contribution to the well-known pre-

\footnotetext{
${ }^{1}$ We shall work with $c=k_{\mathrm{B}}=1$, but we explicitly show the Newton constant $G_{\mathrm{N}}$ and the Planck constant $\hbar$. We also recall that the Planck length is defined as $\ell_{\mathrm{p}}{ }^{2}=G_{\mathrm{N}} \hbar / c^{3}$, the Planck energy as $\mathcal{E}_{\mathrm{p}} \ell_{\mathrm{p}}=\hbar c / 2$, and the Planck mass as $m_{\mathrm{p}}=\mathcal{E}_{\mathrm{p}} / c^{2}$, so that $G_{\mathrm{N}}=\ell_{\mathrm{p}} / 2 m_{\mathrm{p}}$ and $\hbar=$ $2 \ell_{\mathrm{p}} m_{\mathrm{p}}$.
} 
cession of $43 \% /$ century due to general relativity (GR). He then compares this global result with the observational data, and the very accurate agreement between the GR prediction and observations leaves Chang not much room for possible extra contributions to the precession. In fact, he obtains the tremendously small bound $\beta \lesssim 10^{-66}$. A problem with this approach is that a GUP-deformed Newtonian mechanics is simply superposed linearly to the usual GR theory. One may argue that a modification of GR at order $\beta$ should likewise be considered, but this is, however, omitted in Ref. [24]. In other words, it is not clear why the two structures, GR and GUP-modified Newtonian mechanics, should coexist independently, and why the two different precession errors add into a final single precession angle. Most important, as a matter of fact, in the limit $\beta \rightarrow 0$, Ref. [24] recovers only the Newtonian mechanics but not GR, and GR corrections must be added as an extra structure. Clearly, the physical relevance of this approach and the bound that follows for $\beta$ remain therefore questionable.

Finally, a third group of works on the evaluation of $\beta$ contains, for example, papers by Ghosh [27] and Pramanik $[28,29]$. They use a covariant formalism, first defined in Minkowski space, with the metric $\eta_{\mu \nu}=\operatorname{diag}(1,-1$, $-1,-1)$, which can easily be generalized to curved spacetimes via the standard procedure $\eta_{\mu \nu} \rightarrow g_{\mu \nu}$. These papers should, however, be considered as belonging to the second group. In fact, a closer look reveals that they also start from a deformation of classical Poisson brackets, although posited in covariant form. From the deformed covariant Poisson brackets, they obtain interesting consequences, like a $\beta$ deformed geodesic equation, which leads to a violation of the equivalence principle. They do not deform the field equations or the metric. In Appendix A, however, we show that this violation of the equivalence principle is completely due to the postulate of deformed Poisson brackets and has nothing to do with the covariant formalism, or with a deformation of the GR field equations or solutions, or of the geodesic equation. Nonetheless, the Ghosh-Pramanik formalism remains covariant when $\beta \rightarrow 0$ and reproduces standard GR results in the limit $\beta \rightarrow 0$ (this differs, in general, from the results obtained by papers in the second group). The role of the equivalence principle in the GUP context has been discussed also by Tkachuk in Ref. [30], where a correct formulation of the GUP when applied to composite systems is introduced. Also Tkachuk, however, adopts deformed Poisson brackets, therefore automatically inducing a violation of the equivalence principle.

The novelties of our approach, when compared with the previous ones, are many and various. The main point is to start directly from a quantum mechanical effect, the Hawking evaporation, for which the GUP is necessarily relevant, rather than postulating specific representations of canonical operators or modifications of the classical equations of motion. We connect the deformation of the Schwarzschild metric directly to the uncertainty relation, without relying on a specific representation of commutators. We leave the Poisson brackets and classical Newtonian mechanics untouched, and recover $\mathrm{GR}$, and standard quantum mechanics, in the limit $\beta \rightarrow 0$. In particular, we preserve the equivalence principle, and the equation of motion of a test particle is still given by the standard geodesic equation. In the present work, this is obtained by deforming a specific solution of the standard GR field equations, namely the Schwarzschild metric. In Appendix B, we display a non-relativistic analog of this procedure. A further, more profound, step in this direction would be to formulate from scratch the deformed field equations of GR, not just assume a deformed solution (as we did), or a deformed kinematics (as Chang, Ghosh, etc. did). This task is left for future developments.

\section{Deforming the Schwarzschild metric}

In this section, we start from a well-known way of deriving the Hawking temperature directly from the metric of a black hole, and then we show how the GUP modifies the Hawking temperature. These two steps will pave the road to a deformation of the Schwarzschild metric, constructed so as to reproduce the GUP-modified Hawking temperature.

\subsection{Standard mass-temperature relation}

We consider here a space-time with a metric that locally has the form

$$
\mathrm{d} s^{2}=g_{\mu \nu} \mathrm{d} x^{\mu} \mathrm{d} x^{\nu}=F(r) \mathrm{d} t^{2}-F(r)^{-1} \mathrm{~d} r^{2}-r^{2} \mathrm{~d} \Omega^{2},
$$

where $d \Omega^{2}=d \theta^{2}+\sin ^{2} \theta d \phi^{2}$. For the typical cases we shall consider later on, one has

$$
F(r)=1-\frac{2 G_{\mathrm{N}} M}{r}+\frac{G_{\mathrm{N}} Q^{2}}{r^{2}}+\Lambda r^{2},
$$

however, we do not require any specific form for $F(r)$ for the moment. Note that the time-like coordinate is chosen as $x^{0}=t$, the parameters $M$ (mass), $Q$ (electric charge), $\Lambda$ (cosmological constant, up to a factor) are real and continuous, with $\Lambda<0$ corresponding to a de Sitter space-time, and $\Lambda>0$ to an anti de Sitter space-time. The horizons (if any), are located at the positive zeros of the function $F(r)$ (see, for example, Ref. [31]).

We shall loosely follow the derivation in Ref. [32]. Suppose $r=r_{\mathrm{H}}$ is an horizon, so that $F\left(r_{\mathrm{H}}\right)=0$, and consider $r \geq r_{\mathrm{H}}$. After the Wick rotation $t \rightarrow i \tau$ the metric reads

$\mathrm{d} s^{2}=-\left[F(r) \mathrm{d} \tau^{2}+F(r)^{-1} \mathrm{~d} r^{2}+r^{2} \mathrm{~d} \Omega^{2}\right]$. 
In the region just outside the horizon, $r \gtrsim r_{H}$, we perform the coordinate transformation $(\tau, r) \rightarrow(\alpha, R)$ defined by

$$
\left\{\begin{array}{l}
R \mathrm{~d} \alpha=F(r)^{1 / 2} \mathrm{~d} \tau \\
\mathrm{d} R=F(r)^{-1 / 2} \mathrm{~d} r
\end{array}\right.
$$

so that the Euclidean metric becomes

$d s^{2}=-\left[R^{2} \mathrm{~d} \alpha^{2}+\mathrm{d} R^{2}+r^{2}(R) \mathrm{d} \Omega^{2}\right]$.

The first two terms in $\mathrm{d} s^{2}$ represent the length element squared of flat two-dimensional Euclidean space in polar coordinates. The Euclidean time $\tau$ is therefore proportional to the polar angle $\alpha$. Now, denote the period of $\tau$ by $\Theta$ (the period of $\alpha$ is of course $2 \pi$ ). From the second equation we see that $R$ is a function of $r$ only. Therefore, integrating the first of the Eq. (6) over a full period, we get

$R(r) \int_{0}^{2 \pi} \mathrm{d} \alpha=\sqrt{F(r)} \int_{0}^{\Theta} \mathrm{d} \tau$,

that is,

$2 \pi R(r)=\Theta \sqrt{F(r)}$.

We are interested in what happens just outside the horizon, therefore we can expand $F(r)$ around $r_{\mathrm{H}}$. Namely, for $\left(r-r_{\mathrm{H}}\right)$ small we obtain

$$
\begin{aligned}
\sqrt{F(r)} & =\left[F\left(r_{\mathrm{H}}\right)+F^{\prime}\left(r_{\mathrm{H}}\right)\left(r-r_{\mathrm{H}}\right)+\cdots\right]^{1 / 2} \\
& \simeq \sqrt{F^{\prime}\left(r_{\mathrm{H}}\right)} \sqrt{r-r_{\mathrm{H}}} .
\end{aligned}
$$

Equation (9) thus becomes

$2 \pi R(r) \simeq \Theta \sqrt{F^{\prime}\left(r_{\mathrm{H}}\right)} \sqrt{r-r_{\mathrm{H}}}$.

The second of Eq. (6) likewise becomes

$\mathrm{d} R(r) \simeq \frac{\mathrm{d} r}{\sqrt{F^{\prime}\left(r_{\mathrm{H}}\right)} \sqrt{r-r_{\mathrm{H}}}}$,

which yields

$R(r) \simeq \frac{2}{\sqrt{F^{\prime}\left(r_{\mathrm{H}}\right)}} \sqrt{r-r_{\mathrm{H}}}$.

This, together with Eq. (11), implies

$\Theta=\frac{4 \pi}{F^{\prime}\left(r_{\mathrm{H}}\right)}$.

Now, $\Theta$ is the period of the Euclidean time, which means, by the general principles of QFT, that a quantized scalar field outside the horizon lives in a heat bath with temperature $T=\hbar \Theta^{-1}$. To conclude, the temperature of the black hole horizon as seen by a distant observer is in general given by

$T=\hbar \frac{F^{\prime}\left(r_{\mathrm{H}}\right)}{4 \pi}$.

In particular, for a Schwarzschild black hole the function $F(r)$ is given by $Q=\Lambda=0$ in Eq. (3) above, the horizon is at $r_{H}=2 G_{\mathrm{N}} M$, and we get
$T_{\mathrm{H}}=\frac{\hbar}{8 \pi G_{\mathrm{N}} M}$,

which is the well-known Hawking temperature.

\subsection{GUP-modified mass-temperature relation}

The most common form of deformation of the Heisenberg uncertainty relation (and the form of GUP that we are going to study in this paper) is without doubt the following:

$$
\begin{aligned}
\Delta x \Delta p & \geq \frac{\hbar}{2}\left(1+\beta \frac{4 \ell_{\mathrm{p}}^{2}}{\hbar^{2}} \Delta p^{2}\right) \\
& =\frac{\hbar}{2}\left[1+\beta\left(\frac{\Delta p}{m_{\mathrm{p}}}\right)^{2}\right],
\end{aligned}
$$

which, for mirror-symmetric states (with $\langle\hat{p}\rangle^{2}=0$ ), can be equivalently written in terms of commutators as

$[\hat{x}, \hat{p}]=i \hbar\left[1+\beta\left(\frac{\hat{p}}{m_{\mathrm{p}}}\right)^{2}\right]$,

since $\Delta x \Delta p \geq(1 / 2)|\langle[\hat{x}, \hat{p}]\rangle|$. The dimensionless parameter $\beta$ is usually assumed to be of order one, in the most common quantum gravity formulations.

We now give here a derivation of the mass-temperature relation starting directly from the uncertainty relations. As is well known from the argument of the Heisenberg microscope [33], the size $\delta x$ of the smallest detail of an object, theoretically detectable with a beam of photons of energy $E$, is roughly given by

$\delta x \simeq \frac{\hbar}{2 E}$,

since larger and larger energies are required to explore smaller and smaller details. From the uncertainty relation (17), we see that the GUP version of the standard Heisenberg formula (19) is

$\delta x \simeq \frac{\hbar}{2 E}+2 \beta \ell_{\mathrm{p}}^{2} \frac{E}{\hbar}$.

which relates the (average) wavelength of a photon to its energy $E$. (The standard dispersion relation $E=p c$ is assumed.) Conversely, with the relation (20) one can compute the energy $E$ of a photon with a given (average) wavelength $\lambda \simeq \delta x$. Following the arguments of Refs. [34-40], we can consider an ensemble of unpolarized photons of Hawking radiation just outside the event horizon of a Schwarzschild black hole. From a geometrical point of view, it is easy to see that the position uncertainty of such photons is of the order of the unmodified Schwarzschild radius,

$R_{\mathrm{H}}=2 G_{\mathrm{N}} M$.

An equivalent argument comes from considering the average wavelength of the Hawking radiation, which is of the order 
of the geometrical size of the hole. In both cases we can estimate the uncertainty in photon position as

$\delta x \simeq 2 \mu R_{\mathrm{H}}$,

where the proportionality constant $\mu$ is of order unity and will be fixed soon. According to the equipartition principle, the average energy $E$ of unpolarized photons of the Hawking radiation is simply related with their temperature by

$E=T$.

Inserting Eqs. (22) and (23) into the formula (20), we have

$4 \mu G_{\mathrm{N}} M \simeq \frac{\hbar}{2 T}+2 \beta G_{\mathrm{N}} T$.

In order to fix $\mu$, we consider the semiclassical limit, $\beta \rightarrow$ 0 , and require that Eq. (24) predicts the standard semiclassical Hawking temperature (16), that is, $T(\beta \rightarrow 0)=T_{\mathrm{H}}$. This fixes $\mu=\pi$, so that we have

$M=\frac{\hbar}{8 \pi G_{\mathrm{N}} T}+\beta \frac{T}{2 \pi}$.

This is the mass-temperature relation predicted by the GUP for a Schwarzschild black hole. Of course, this relation can easily be inverted,

$T=\frac{\pi}{\beta}\left(M-\sqrt{M^{2}-\frac{\beta}{\pi^{2}} m_{\mathrm{p}}^{2}}\right)$,

where we used $\hbar / G_{\mathrm{N}}=4 m_{\mathrm{p}}{ }^{2}$. Since, however, the term proportional to $\beta$ is small, especially for solar mass black holes with $M \gg m_{\mathrm{p}}$, we can expand in powers of $\beta$, namely

$T=T_{\mathrm{H}}\left(1+\frac{\beta m_{\mathrm{p}}^{2}}{4 \pi^{2} M^{2}}+\cdots\right)$.

To zero order in $\beta$, we recover the usual Hawking formula (16). Already from this we can extract an interesting estimate of $\beta$, since the previous expansion is valid only if

$\frac{\beta m_{\mathrm{p}}^{2}}{4 \pi^{2} M^{2}} \ll 1$

which means $\beta \ll 1.3 \times 10^{78}$ for a solar mass black hole with $M \simeq 10^{38} m_{\mathrm{p}}$.

Let us note that in this work we assume that the correction induced by the GUP has a thermal character, and therefore it can be cast in the form of a shift of the Hawking temperature. Of course, there are also different approaches, where the corrections do not respect the exact thermality of the spectrum, and thus they need not be reducible to a simple shift of the temperature. An example is the corpuscular model of a black hole of Ref. [41]. In this model, the emission is expected to gain a correction of order $1 / N$, where $N \sim\left(M / m_{\mathrm{p}}\right)^{2}$ is the number of constituents, and it becomes important when the mass $M$ approaches the Planck mass.

\subsection{GUP-modified Schwarzschild metric}

We can legitimately wonder what kind of (deformed) metric would predict a Hawking temperature like the one inferred from the GUP relation (25), for a given $\beta$. Since we are interested only in small corrections to the Hawking formula, we can consider a deformation of the Schwarzschild metric of the kind

$F(r)=1-\frac{2 G_{\mathrm{N}} M}{r}+\varepsilon \frac{G_{\mathrm{N}}^{2} M^{2}}{r^{2}}$,

and we shall look for the lowest order correction in $\varepsilon$. We see that Eq. (29) is actually the simplest mathematical form, if one supposes that the metric can be expanded in powers of $1 / r$. This is nothing else than the well-known Eddington-Robertson expansion of a spherically symmetric metric. Note, however, that, since $R_{\mathrm{H}} / r \sim 10^{-5}$ on the surface of the Sun, the term proportional to $\varepsilon$ can still be considered small even if $\varepsilon$ is relatively large. The horizons are now given by solutions of the equation

$r^{2}-2 G_{\mathrm{N}} M r+\varepsilon G_{\mathrm{N}}^{2} M^{2}=0$.

We choose the root closest to the unmodified Schwarzschild radius (21), namely

$r_{\mathrm{H}}=R_{\mathrm{H}} \frac{1+\sqrt{1-\varepsilon}}{2}$,

which is valid for $\varepsilon \leq 1$ (and possibly negative). Then

$F^{\prime}(r)=\frac{2 G_{\mathrm{N}} M}{r^{2}}-2 \varepsilon \frac{G_{\mathrm{N}}^{2} M^{2}}{r^{3}}$

and

$$
\begin{aligned}
F^{\prime}\left(r_{\mathrm{H}}\right) & =\frac{2}{G_{\mathrm{N}} M} \frac{\sqrt{1-\varepsilon}}{(1+\sqrt{1-\varepsilon})^{2}} \\
& \simeq \frac{1}{R_{\mathrm{H}}}\left(1-\frac{\varepsilon^{2}}{16}+\cdots\right),
\end{aligned}
$$

where the last expansion is valid for $|\varepsilon| \ll 1$. Hence, the temperature predicted by this deformed Schwarzschild metric is

$T(\varepsilon)=\hbar \frac{F^{\prime}\left(r_{\mathrm{H}}\right)}{4 \pi}=\frac{\hbar}{2 \pi G_{\mathrm{N}} M} \frac{\sqrt{1-\varepsilon}}{(1+\sqrt{1-\varepsilon})^{2}}$,

which must coincide with the temperature $T(\beta)$ predicted by Eq. (26), for any given $\beta$. That is, $T(\varepsilon)$ should solve Eq. (25),

$M=\frac{\hbar}{8 \pi G_{\mathrm{N}} T(\varepsilon)}+\frac{\beta}{2 \pi} T(\varepsilon)$,

which yields a relation between $\beta$ and $\varepsilon$,

$\beta(\varepsilon)=-\pi^{2} \frac{G_{\mathrm{N}} M^{2}}{\hbar} \frac{\varepsilon^{2}}{1-\varepsilon}$.

For $|\varepsilon| \ll 1$, to the lowest order in $\varepsilon$, we thus get

$\beta=-\frac{\pi^{2} M^{2}}{4 m_{\mathrm{p}}^{2}} \varepsilon^{2}$, 
where we notice that both $\beta$ and $\varepsilon$ are dimensionless. It is now of great interest to observe that Eq. (36) forces us to admit that $\beta<0$, since $\varepsilon \leq 1$. Although quite unexpected, this might be a suggestion of fundamental importance. It seems that a metric is able to reproduce the GUP-deformed Hawking temperature only if the deforming parameter $\beta$ is negative. We already encountered a situation like this when we studied the uncertainty relation formulated on a crystal lattice [42]. This could be a further hint that the physical space-time has actually a lattice or granular structure at the level of the Planck scale.

\section{Light deflection by deformed Schwarzschild metric}

Having established a connection between the GUP parameter $\beta$ and the deformation $\varepsilon$ of the Schwarzschild metric, we are now in a position to compute the physical (possible observable) consequences of such a deformed metric. To begin with, we examine the unbound orbits around a massive body, namely the light deflection by the Sun. Our treatment roughly follows that of Ref. [43].

With reference to Fig. 1, we consider a polar coordinate system centered in the Sun, with $(\phi, r)$ labeling the position of the incoming photon; $\phi=\phi(r)$ describes the photon orbit, $R_{\odot}$ is the radius of the Sun, $r_{0}$ the minimum distance between the photon and Sun. The photon orbit is thus described by

$\phi(r)-\phi(\infty)=\int_{\infty}^{r} \frac{1}{r}\left[\left(\frac{r}{r_{0}}\right)^{2} F\left(r_{0}\right)-F(r)\right]^{-1 / 2} \mathrm{~d} r$.

The global deflection angle of the orbit from a straight line is just twice its change from $\infty$ to $r_{0}$, of course minus $\pi$,

$\Delta \phi \equiv 2\left|\phi\left(r_{0}\right)-\phi(\infty)\right|-\pi$.

This integral can be evaluated exactly by using elliptic integrals, which, however, can only be numerically computed by expanding in a suitable small parameter. It is both easier and more useful to expand before integrating. Care must be taken in choosing the right small parameter, which will also help in identifying the finite part of the integral. Here physical intuition comes in for help. In fact, if the central body had a negligible mass, then $F(r) \rightarrow 1$ (the Minkowskian limit), and the trajectory of the photon would be a (almost) straight line. Departure from a straight line increases as the central mass $M \sim R_{\mathrm{H}}$ increases, as well as if the minimum distance from the source $r_{0}$ decreases. The right parameter by which to expand the integral (38) is thus the ratio $R_{\mathrm{H}} / r_{0}$.

Now, the argument of the integral can be written as

$$
\begin{aligned}
& \frac{1}{r}\left[\left(\frac{r}{r_{0}}\right)^{2} F\left(r_{0}\right)-F(r)\right]^{-1 / 2} \\
& \quad=\frac{1}{r}\left[\left(\frac{r}{r_{0}}\right)^{2}-1\right]^{-1 / 2} \times\left[F\left(r_{0}\right)+\frac{F\left(r_{0}\right)-F(r)}{\left(r / r_{0}\right)^{2}-1}\right]^{-1 / 2},
\end{aligned}
$$

and we find, to first order in $\varepsilon$ and to the second order in $R_{\mathrm{H}} / r_{0}$,

$$
\begin{aligned}
& {\left[F\left(r_{0}\right)+\frac{F\left(r_{0}\right)-F(r)}{\left(r / r_{0}\right)^{2}-1}\right]^{-1 / 2}} \\
& =\left[1-\frac{2 G_{\mathrm{N}} M}{r_{0}}+\varepsilon \frac{G_{\mathrm{N}}^{2} M^{2}}{r_{0}^{2}}-\frac{2 G_{\mathrm{N}} M r_{0}}{r\left(r+r_{0}\right)}+\varepsilon \frac{G_{\mathrm{N}}^{2} M^{2}}{r^{2}}\right]^{-1 / 2} \\
& \simeq 1+\frac{G_{\mathrm{N}} M}{r_{0}}+(3-\varepsilon) \frac{G_{\mathrm{N}}^{2} M^{2}}{2 r_{0}^{2}}+\frac{G_{\mathrm{N}} M r_{0}}{r\left(r+r_{0}\right)}\left(1+\frac{3 G_{\mathrm{N}} M}{r_{0}}\right) \\
& \quad-\varepsilon \frac{G_{\mathrm{N}}^{2} M^{2}}{2 r^{2}}+\frac{3 G_{\mathrm{N}}^{2} M^{2} r_{0}^{2}}{2 r^{2}\left(r+r_{0}\right)^{2}},
\end{aligned}
$$

where we employed the expansion $(1+\delta)^{-1 / 2} \simeq 1-\frac{\delta}{2}+$ $\frac{3}{8} \delta^{2}$. The integral (38) now becomes

$\phi(r)-\phi(\infty) \simeq A+B+C+D$,

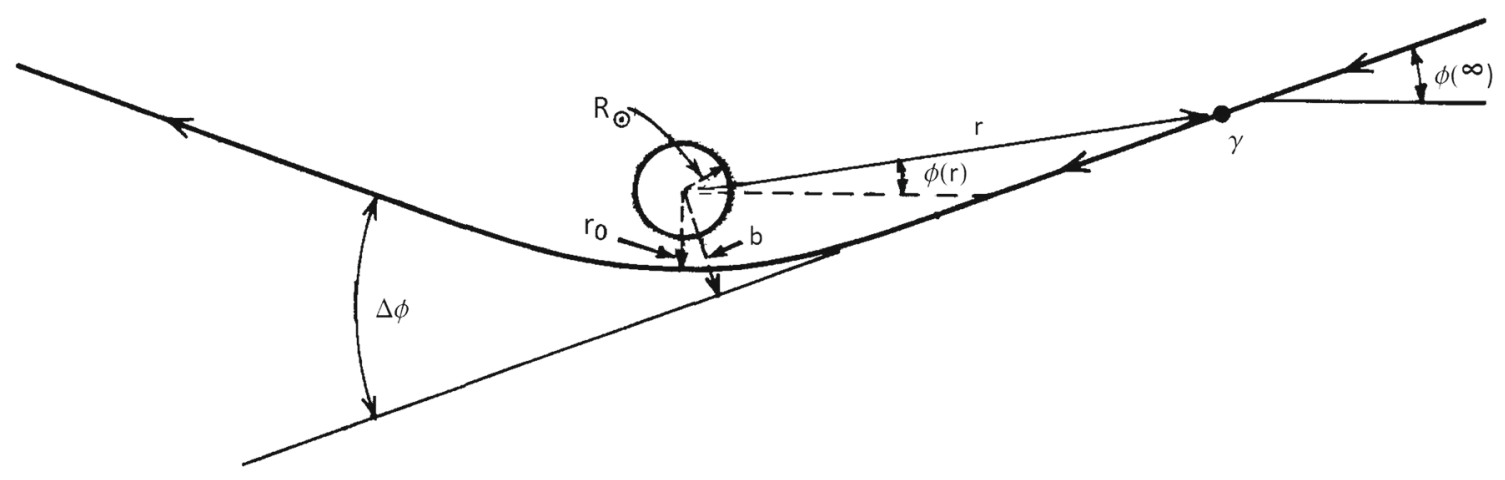

Fig. 1 Deflection of light by the Sun (refer to the text) 
where

$$
\begin{aligned}
A= & \int_{\infty}^{r} \frac{1}{r}\left[\left(\frac{r}{r_{0}}\right)^{2}-1\right]^{-1 / 2} \\
& \times\left[1+\frac{G_{\mathrm{N}} M}{r_{0}}+(3-\varepsilon) \frac{G_{\mathrm{N}}^{2} M^{2}}{2 r_{0}^{2}}\right] \mathrm{d} r \\
B= & \int_{\infty}^{r} \frac{1}{r}\left[\left(\frac{r}{r_{0}}\right)^{2}-1\right]^{-1 / 2} \frac{G_{\mathrm{N}} M r_{0}}{r\left(r+r_{0}\right)}\left(1+\frac{3 G_{\mathrm{N}} M}{r_{0}}\right) \mathrm{d} r \\
C= & -\int_{\infty}^{r} \frac{1}{r}\left[\left(\frac{r}{r_{0}}\right)^{2}-1\right]^{-1 / 2} \frac{\varepsilon G_{\mathrm{N}}^{2} M^{2}}{2 r^{2}} \mathrm{~d} r
\end{aligned}
$$

and

$D=\int_{\infty}^{r} \frac{1}{r}\left[\left(\frac{r}{r_{0}}\right)^{2}-1\right]^{-1 / 2} \frac{3 G_{\mathrm{N}}^{2} M^{2} r_{0}^{2}}{2 r^{2}\left(r+r_{0}\right)^{2}} \mathrm{~d} r$.

These integrals are all elementary, and we obtain

$$
\begin{aligned}
\phi(r) & -\phi(\infty) \\
\simeq & -\left[1+\frac{G_{\mathrm{N}} M}{r_{0}}+(3-\varepsilon) \frac{G_{\mathrm{N}}^{2} M^{2}}{2 r_{0}^{2}}\right] \arcsin \left(\frac{r_{0}}{r}\right) \\
+ & \frac{G_{\mathrm{N}} M}{r_{0}}\left(1+\frac{3 G_{\mathrm{N}} M}{r_{0}}\right) \\
\times & {\left[\sqrt{1-\left(\frac{r_{0}}{r}\right)^{2}}\left(1+\frac{r}{r+r_{0}}\right)+\arcsin \left(\frac{r_{0}}{r}\right)-2\right] } \\
- & \frac{\varepsilon G_{\mathrm{N}}^{2} M^{2}}{2 r_{0}^{2}}\left[\frac{r_{0}}{2 r} \sqrt{1-\left(\frac{r_{0}}{r}\right)^{2}}-\frac{1}{2} \arcsin \left(\frac{r_{0}}{r}\right)\right] \\
- & \frac{3 G_{\mathrm{N}}^{2} M^{2}}{2 r_{0}^{2}}\left[\sqrt{1-\left(\frac{r_{0}}{r}\right)^{2}}\right] \\
\times & \left(2-\frac{r_{0}}{2 r}-\frac{r^{2}}{3\left(r+r_{0}\right)^{2}}+\frac{11 r}{3\left(r+r_{0}\right)}\right) \\
+ & \left.\left.\frac{7}{2} \arcsin ^{2} \frac{r_{0}}{r}\right)-\frac{16}{3}\right]
\end{aligned}
$$

and finally

$\Delta \phi \simeq \frac{2 R_{\mathrm{H}}}{r_{0}}+\frac{R_{\mathrm{H}}^{2}}{16 r_{0}^{2}}(15 \pi-16-3 \pi \varepsilon)$,

which shows that ours is indeed an expansion in $R_{\mathrm{H}} / r_{0}$. Notice also that the term of second order in $R_{\mathrm{H}} / r_{0}$ does not vanish when $\varepsilon \rightarrow 0$, since we properly expanded the integrand to this order (on this expansion, see also Ref. [44]).

Our result can now be compared with the deflection angle of a light ray (or a photon) just grazing the Sun surface, which is usually given in the form

$\Delta \phi=\frac{1}{2}(1+\gamma) \frac{2 R_{\mathrm{H}}}{r_{0}}$, where $r_{0}=R_{\odot}$ and $R_{\mathrm{H}}=2 G_{\mathrm{N}} M_{\odot}$. The best measurements presently available for the parameter $\gamma$ from the light bending close to the surface of the Sun are given by the development of the very-long-baseline radio interferometry (VLBI, see Ref. [45]). A 2004 analysis of almost 2 million VLBI observations of 541 radio sources, made by 87 VLBI sites, yielded $\gamma-1 \simeq(-1.7 \pm 4.5) \times 10^{-4}$ [46]. A 2009 analysis updated through 2008 data yielded $\gamma-1 \simeq(-1.6 \pm 1.5) \times 10^{-4}$ [47]. Good measurements were also performed by the optical astrometry satellite Hipparcos (at the level of $0.1 \%$ ), and significant improvements are expected from the just launched satellite Gaia (see Ref. [48]). On comparing Eq. (44) with (45), we immediately get

$\gamma-1=\frac{G_{\mathrm{N}} M}{8 r_{0}}(15 \pi-16-3 \pi \varepsilon)$,

or

$|\gamma-1|=\frac{G_{\mathrm{N}} M}{8 r_{0}}|15 \pi-16-3 \pi \varepsilon| \lesssim 1.6 \times 10^{-4}$.

For $M=M_{\odot}$ and $r_{0}=R_{\odot}$, this means $-60.7<\varepsilon<67.3$, which, together with the mathematical constraint $\varepsilon \leq 1$, gives a range for the $\varepsilon$ values of about

$-65 \lesssim \varepsilon \leq 1$

Assuming the "worst" situation, namely $\varepsilon \simeq-65$, then Eq. (36) gives the upper bound for the GUP parameter,

$|\beta|=\frac{M^{2}}{4 m_{\mathrm{p}}^{2}} \frac{\pi^{2} \varepsilon^{2}}{1-\varepsilon} \lesssim 5.3 \times 10^{78}$,

which is comparable with the limit obtained from general considerations on the validity of the low $\beta$ expansion (28). This not-so-tight bound has to do with the well-known fact that light deflection is not the most precise test of GR (since light deflection is still a "Newtonian" phenomenon, see Ref. [49]). A much better estimation will be obtained in the next section, where the perihelion precession is considered.

\section{Perihelion precession by deformed Schwarzschild metric}

Here, we consider a particle bound in an orbit around a massive body, typically a planet around the Sun. Again, we roughly follow the treatment of Ref. [43]. In Fig. 2 we can see the relevant geometrical parameters for an elliptic orbit in a polar coordinates system, with the radial coordinate $r$ which at aphelion and perihelion takes, respectively, the maximum value $r_{+}$and minimum value $r_{-} ; e$ is the eccentricity, $a$ the semi-major axis, and $L$ the semilatus rectum. These geometrical parameters are related by 


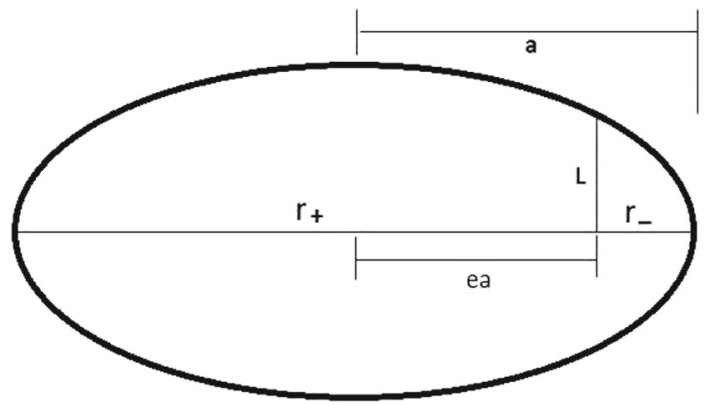

Fig. 2 Elements of the ellipse used in the text for the calculations of precession of planetary orbits

$$
\begin{aligned}
r_{ \pm} & =(1 \pm e) a \\
L & =\left(1-e^{2}\right) a \\
\frac{2}{L} & =\frac{1}{r_{+}}+\frac{1}{r_{-}} .
\end{aligned}
$$

The two relevant constants of motion of the system, $E$ and $J$, can be interpreted, respectively, as the energy per unit mass, $(1-E) / 2$, and an angular momentum per unit mass (see Ref. [43]). The constants $E$ and $J$ can be further expressed as functions of $F\left(r_{-}\right)$and $F\left(r_{+}\right)$. The angle swept out by the position vector when it increases from $r_{-}$to $r$ is then given by the integral

$$
\begin{aligned}
\phi(r) & -\phi\left(r_{-}\right) \\
= & \int_{r_{-}}^{r}\left[\frac{r_{-}^{2}\left(\frac{1}{F(r)}-\frac{1}{F\left(r_{-}\right)}\right)-r_{+}^{2}\left(\frac{1}{F(r)}-\frac{1}{F\left(r_{+}\right)}\right)}{r_{-}^{2} r_{+}^{2}\left(\frac{1}{F\left(r_{+}\right)}-\frac{1}{F\left(r_{-}\right)}\right)}-\frac{1}{r^{2}}\right]^{-1 / 2} \\
& \times \frac{\mathrm{d} r}{r^{2} \sqrt{F(r)}} .
\end{aligned}
$$

The total change in $\phi$ at every lap is just twice the change as $r$ increases from $r_{-}$to $r_{+}$. This would equal $2 \pi$ if the orbit were a closed ellipse, so the total orbital precession in each revolution is given by

$\Delta \phi=2\left|\phi\left(r_{+}\right)-\phi\left(r_{-}\right)\right|-2 \pi$.

As before, the exact value of the integral can be expressed via elliptic functions, but then one has to expand the elliptic functions to obtain useful results. It is much faster to expand before integrating, where, in analogy with the case of light deflection, the small parameter is now given by $R_{\mathrm{H}} / r_{-}$. Since $R_{\mathrm{H}} / r \lesssim R_{\mathrm{H}} / r_{-}$all along the orbit, this also implies that one can expand in $R_{\mathrm{H}} / r$. From the definition of $F(r)$, to second order in $R_{\mathrm{H}} / r$, we have

$\frac{1}{F(r)} \simeq 1+\frac{2 G_{\mathrm{N}} M}{r}+(4-\varepsilon) \frac{G_{\mathrm{N}}^{2} M^{2}}{r^{2}}$,

so the expression in the square brackets inside the integral in Eq. (53) is actually a quadratic function of $1 / r$. Moreover, it vanishes for $r=r_{ \pm}$, and we can therefore write

$$
\begin{aligned}
& \frac{r_{-}^{2}\left(\frac{1}{F(r)}-\frac{1}{F\left(r_{-}\right)}\right)-r_{+}^{2}\left(\frac{1}{F(r)}-\frac{1}{F\left(r_{+}\right)}\right)}{r_{-}^{2} r_{+}^{2}\left(\frac{1}{F\left(r_{+}\right)}-\frac{1}{F\left(r_{-}\right)}\right)}-\frac{1}{r^{2}} \\
& =C\left(\frac{1}{r_{-}}-\frac{1}{r}\right)\left(\frac{1}{r}-\frac{1}{r_{+}}\right),
\end{aligned}
$$

so that the Eq. (53) for the trajectory becomes

$$
\begin{aligned}
& \phi(r)-\phi\left(r_{-}\right) \\
& =\frac{1}{\sqrt{C}} \int_{r_{-}}^{r}\left[\left(\frac{1}{r_{-}}-\frac{1}{r}\right)\left(\frac{1}{r}-\frac{1}{r_{+}}\right)\right]^{-1 / 2} \frac{\mathrm{d} r}{r^{2} \sqrt{F(r)}} .
\end{aligned}
$$

We can find the constant $C$ by considering the limit $r \rightarrow \infty$, and hence $F(r)^{-1} \rightarrow 1$,

$C=\frac{r_{+}^{2} F\left(r_{-}\right)\left[F\left(r_{+}\right)-1\right]-r_{-}^{2} F\left(r_{+}\right)\left[F\left(r_{-}\right)-1\right]}{r_{-} r_{+}\left[F\left(r_{-}\right)-F\left(r_{+}\right)\right]}$.

By inserting the expressions for $F\left(r_{ \pm}\right)$from (29), and recalling Eq. (52) for $L$, with a bit of algebra, we get the exact expression

$C=\left(1-\frac{2 R_{\mathrm{H}}}{L}+\varepsilon \frac{R_{\mathrm{H}}^{2}}{L^{2}}-\varepsilon^{2} \frac{R_{\mathrm{H}}^{3}}{8 a L^{2}}\right)\left(1-\varepsilon \frac{R_{\mathrm{H}}}{2 L}\right)^{-1}$.

We can now expand to second order in $R_{\mathrm{H}} / L$,

$$
\begin{aligned}
C^{-1 / 2} \simeq 1 & +\left(\frac{4-\varepsilon}{2}\right) \frac{G_{\mathrm{N}} M}{L} \\
& +\left(6-3 \varepsilon-\frac{1}{8} \varepsilon^{2}\right) \frac{G_{\mathrm{N}}^{2} M^{2}}{L^{2}},
\end{aligned}
$$

and, to second order in $R_{\mathrm{H}} / r$,

$[F(r)]^{-1 / 2} \simeq 1+\frac{G_{\mathrm{N}} M}{r}+(3-\varepsilon) \frac{G_{\mathrm{N}^{2}} M^{2}}{2 r^{2}}$.

The integral in Eq. (57) is largely simplified if we choose a clever change of variable. Let us consider the closed orbit predicted by Newtonian mechanics. Taking the pole in the direction of the semilatus rectum, the polar equation of the trajectory reads

$\frac{1}{r}=\frac{1}{L}-\frac{e}{L} \sin \psi$,

and this will be our change of variable in the integral (57), with

$\frac{\mathrm{d} r}{r^{2}}=\frac{e}{L} \cos \psi \mathrm{d} \psi$

On recalling Eq. (52) and

$\frac{e}{L}=\frac{1}{2}\left(\frac{1}{r_{-}}-\frac{1}{r_{+}}\right)$ 
we have

$$
\begin{aligned}
& \frac{1}{r_{-}}-\frac{1}{r}=\frac{e}{L}(1+\sin \psi), \\
& \frac{1}{r}-\frac{1}{r_{+}}=\frac{e}{L}(1-\sin \psi),
\end{aligned}
$$

and

$$
\left(\frac{1}{r_{-}}-\frac{1}{r}\right)\left(\frac{1}{r}-\frac{1}{r_{+}}\right)=\frac{e^{2}}{L^{2}} \cos ^{2} \psi .
$$

When $r=r_{-}$, then $\psi=-\pi / 2$. Inserting everything back into Eq. (57), to second order in $R_{\mathrm{H}} / r$, we get

$$
\begin{aligned}
& \phi(r)-\phi\left(r_{-}\right) \\
& \simeq\left[1+\left(\frac{4-\varepsilon}{2}\right) \frac{G_{\mathrm{N}} M}{L}+\left(6-3 \varepsilon-\frac{1}{8} \varepsilon^{2}\right) \frac{G_{\mathrm{N}}^{2} M^{2}}{L^{2}}\right] \\
& \quad \times \int_{-\pi / 2}^{\psi}\left[1+\frac{G_{\mathrm{N}} M}{L}(1-e \sin \psi)\right. \\
& \left.\quad+(3-\varepsilon) \frac{G_{\mathrm{N}}^{2} M^{2}}{2 L^{2}}(1-e \sin \psi)^{2}\right] \mathrm{d} \psi .
\end{aligned}
$$

At the aphelion $r=r_{+}$and $\psi=\pi / 2$. The computation of the integral to the same order in $R_{\mathrm{H}} / r$ is elementary and yields

$$
\begin{aligned}
& \phi\left(r_{+}\right)-\phi\left(r_{-}\right) \\
& \simeq\left[1+\left(\frac{4-\varepsilon}{2}\right) \frac{G_{\mathrm{N}} M}{L}+\left(6-3 \varepsilon-\frac{1}{8} \varepsilon^{2}\right) \frac{G_{\mathrm{N}}^{2} M^{2}}{L^{2}}\right] \\
& \quad \times \pi\left[1+\frac{G_{\mathrm{N}} M}{L}+(3-\varepsilon) \frac{G_{\mathrm{N}}^{2} M^{2}}{2 L^{2}}\left(1+\frac{e^{2}}{2}\right)\right] \\
& \simeq \pi\left[1+\left(\frac{6-\varepsilon}{2}\right) \frac{G_{\mathrm{N}} M}{L}+\frac{G_{\mathrm{N}}^{2} M^{2}}{L^{2}} N(\varepsilon, e)\right],
\end{aligned}
$$

where

$N(\varepsilon, e)=\frac{1}{2}\left[19-8 \varepsilon+(3-\varepsilon) \frac{e^{2}}{2}-\frac{\varepsilon^{2}}{4}\right]$.

Finally the total precession after a single lap is given by

$\Delta \phi \simeq 2 \pi\left(\frac{6-\varepsilon}{2}\right) \frac{G_{\mathrm{N}} M}{L}+2 \pi \frac{G_{\mathrm{N}}^{2} M^{2}}{L^{2}} N(\varepsilon, e)$.

In particular, we note that, to first order in $R_{\mathrm{H}} / L$, we can write

$\Delta \phi \simeq \frac{6 \pi G_{\mathrm{N}} M}{L}\left(1-\frac{\varepsilon}{6}\right)$

which, of course, reproduces the usual GR prediction in the limit $\varepsilon \rightarrow 0$. This relation should now be compared with known observational data.

\subsection{Solar system data}

The perihelion precession for Mercury is by far the best known and measured GR precession in the Solar system.
Referring to Ref. [45] for the latest most accurate and comprehensive data, we can report the relation

$\langle\dot{\omega}\rangle=\frac{6 \pi G_{\mathrm{N}} M}{L}\left[\frac{1}{3}(2+2 \gamma-\bar{\beta})+3 \times 10^{-4} \frac{J_{2}}{10^{-7}}\right]$,

where $\langle\dot{\omega}\rangle$ is the measured perihelion shift, $J_{2}$ a dimensionless measure of the quadrupole moment of the Sun, and $\gamma$ and $\bar{\beta}$ are the usual Eddington-Robertson expansion parameters. The latest data from helioseismology give $J_{2}=(2.2 \pm 0.1) \times 10^{-7}$. The measured perihelion shift of Mercury is known very accurately: after the perturbing effects of other planets have been accounted for, the excess shift is known to about $0.1 \%$ from radar observations of Mercury between 1966 and 1990 [50]. The solar oblateness effect due to the quadrupole moment is then smaller than the observational error, so it can be neglected. Substituting standard orbital elements and physical constants for Mercury and the Sun, we obtain

$\langle\dot{\omega}\rangle=\left(1+\frac{2 \gamma-\bar{\beta}-1}{3}\right) 42.98 \% /$ century,

where we can place a bound of $|2 \gamma-\bar{\beta}-1| \lesssim 3 \times 10^{-3}$.

Comparing with $\Delta \phi$ from Eq. (72), we get

$|\varepsilon| \lesssim 6 \times 10^{-3}$

which, on replacing in Eq. (36), yields the lower bound

$|\beta|=\frac{M^{2}}{4 m_{\mathrm{p}}^{2}} \frac{\pi^{2} \varepsilon^{2}}{1-\varepsilon} \lesssim 3 \times 10^{72}$.

We can also consider the most recent data from the Messenger spacecraft [51], which orbited Mercury in 2011-2013, and improved very much the knowledge of its orbit. Then we can push this bound even lower, to $|2 \gamma-\bar{\beta}-1| \lesssim 7.8 \times$ $10^{-5}$, although the knowledge of $J_{2}$ would have to improve simultaneously. If just the error in $|2 \gamma-\bar{\beta}-1|$ were taken into account, this would imply

$|\varepsilon|=2|2 \gamma-\bar{\beta}-1| \lesssim 1.56 \times 10^{-4}$,

and therefore

$|\beta| \lesssim 2 \times 10^{69}$

But of course this limit should not be considered completely reliable in this contest, since the less accurate bound on $J_{2}$ cannot be brutally neglected, at least in principle. As expected, we gain here at least six orders of magnitude, showing once again that the perihelion shift is one of the most precise tests of GR, a true GR effect not present at all in Newtonian gravity (as is well known). We can, however, try to put this limit on a firmer ground by looking for even larger effects of this kind. Where? Of course, in binary pulsars! 
Table 1 Orbital parameters of PRS B 1913+16 [52]. Figures in parentheses represent estimated uncertainties in the last quoted digit

\begin{tabular}{ll}
\hline Parameter & Value \\
\hline$e$ (eccentricity) & $0.6171334(5)$ \\
$P_{\mathrm{b}}$ (days) (orbital period) & $0.322997448911(4)$ \\
$\langle\dot{\omega}\rangle($ deg/year) (periastron shift) & $4.226598(5)$ \\
$\gamma(\mathrm{s})$ (time dilation, gravitational redshift) & $4.2992(8) \times 10^{-3}$ \\
$\dot{P}_{\mathrm{b}}(\mathrm{s} / \mathrm{s})$ (orbital period decay) & $-2.423(1) \times 10^{-12}$ \\
\hline
\end{tabular}

\subsection{Pulsar PRS B 1913+16 data}

Clearly, binary pulsars are very good candidates for measurements of periastron shifts. Among the known pulsar systems, the best tested pair is the pulsar PRS B 1913+16. Discovered in 1974 by Hulse and Taylor, this system has become, after 40 years of observations, one of the most reliable celestial laboratories for precise GR measurements. For example, the prediction of GR for the period decay rate due to emission of gravitational waves coincides with the measured value up to an error on the 14th decimal figure.

The state of the art on this system is described in Ref. [52]. In Table 1, we report the orbital parameters of interest for us. The parameters $e$ and $P_{\mathrm{b}}$ are called Keplerian parameters, since they are well-defined quantities also in the Newtonian theory. On the contrary, $\langle\dot{\omega}\rangle, \gamma, \dot{P}_{\mathrm{b}}$ are known as DamourDeruelle post-Keplerian parameters [53], quantities typically well defined in GR only. In Ref. [54], Taylor and Weisberg have shown that each GR post-Keplerian parameter can be expressed in terms of the Keplerian parameters and of the unknown masses of the pulsar and its companion, $m_{1}, m_{2}$. In fact,

$$
\begin{aligned}
\langle\dot{\omega}\rangle & =3 G_{\mathrm{N}}{ }^{2 / 3} c^{-2}\left(P_{\mathrm{b}} / 2 \pi\right)^{-5 / 3}\left(1-e^{2}\right)^{-1}\left(m_{1}+m_{2}\right)^{2 / 3} \\
& =2.113323(2)\left[\frac{\left(m_{1}+m_{2}\right)}{M_{\odot}}\right]^{2 / 3} \operatorname{deg} \operatorname{year}^{-1}, \\
\gamma= & G_{\mathrm{N}}{ }^{2 / 3} c^{-2} e\left(P_{\mathrm{b}} / 2 \pi\right)^{1 / 3} m_{2} \\
& \times\left(m_{1}+2 m_{2}\right)\left(m_{1}+m_{2}\right)^{-4 / 3} \\
= & 0.002936679(2)\left[\frac{m_{2}\left(m_{1}+2 m_{2}\right)\left(m_{1}+m_{2}\right)^{-4 / 3}}{M_{\odot}^{2 / 3}}\right] \mathrm{s},
\end{aligned}
$$

$$
\begin{aligned}
\dot{P}_{\mathrm{b}}= & -\frac{192 \pi G_{\mathrm{N}}^{5 / 3}}{5 c^{5}}\left(\frac{P_{\mathrm{b}}}{2 \pi}\right)^{-5 / 3} \times\left(1+\frac{73}{24} e^{2}+\frac{37}{96} e^{4}\right) \\
& \left(1-e^{2}\right)^{-7 / 2} m_{1} m_{2}\left(m_{1}+m_{2}\right)^{-1 / 3} \\
= & -1.699451(8) \times 10^{-12}\left[\frac{m_{1} m_{2}\left(m_{1}+m_{2}\right)^{-1 / 3}}{M_{\odot}^{5 / 3}}\right] .
\end{aligned}
$$

To compute the numerical coefficients, in the second line of each equation we have substituted values for $P_{\mathrm{b}}$ and $e$ from Table 1, and we have used the constants $G_{\mathrm{N}} M_{\odot} / c^{3}=$ $4.925490947 \times 10^{-6} \mathrm{~s}$ and 1 Julian year $=86400 \times 365.25 \mathrm{~s}$. The figures in parentheses represent uncertainties in the last quoted digit, determined by propagating the uncertainties listed in Table 1. In each case, the errors are dominated by the experimental uncertainty in orbital eccentricity, $e$. Furthermore, we note that the analytical expression for the periastron angular velocity $\langle\dot{\omega}\rangle$, given above by Taylor and Weisberg, can be obtained from the standard GR prediction for the shift-per-lap $6 \pi G_{\mathrm{N}} M / L$, simply by dividing this for the period $P_{\mathrm{b}}$, and of course with $M=m_{1}+m_{2}$ (other quantities being expressed as a function of $P_{\mathrm{b}}$ and $e$ ).

To get $\langle\dot{\omega}\rangle^{\mathrm{GR}}$, the GR theoretical prediction of the periastron shift, the strategy is the following: first, insert the observational values for $\gamma$ and $\dot{P}_{\mathrm{b}}$ from Table 1 into Eqs. (80) and (81), and solve for $m_{1}, m_{2}$. Then substitute the values of $m_{1}$, $m_{2}$ thus found into Eq. (79) to compute $\langle\dot{\omega}\rangle^{\mathrm{GR}}$, and compare this prediction with the observed value of $\langle\dot{\omega}\rangle$ Obs again given in Table 1. The relative error can then be defined as

$\tilde{\varepsilon}=\frac{\langle\dot{\omega}\rangle^{\mathrm{Obs}}-\langle\dot{\omega}\rangle^{\mathrm{GR}}}{\langle\dot{\omega}\rangle^{\mathrm{GR}}}$,

that is, $\langle\dot{\omega}\rangle^{\mathrm{GR}}(1+\tilde{\varepsilon})=\langle\dot{\omega}\rangle^{\mathrm{Obs}}$, and, on comparing with $\Delta \phi$ in Eq. (72), we get $|\varepsilon|=6|\tilde{\varepsilon}|$.

There is one further issue we should care of: measurements are now so precise that the observed value of $\dot{P}_{\mathrm{b}}$ in Table 1 should be corrected for the relative acceleration between the pulsar reference frame and the solar system center-of-mass frame (see Ref. [55]). Such a relative acceleration is mainly due to the fact that the pulsar and our solar system are located in different arms of our Galaxy, at different distances from the galactic center. The small additional kinematic contribution to the observed $\dot{P}_{\mathrm{b}}$ is

$\Delta \dot{P}_{\mathrm{b}, \mathrm{gal}}=(-0.027 \pm 0.005) \times 10^{-12}$.

We can solve the system of the two Eqs. (80) and (81) for the two unknowns $m_{1}$ and $m_{2}$. The best result is obtained by combining, from Table 1 , the lower bound for $\dot{P}_{\mathrm{b}}=$ $(-2.423-0.001) \times 10^{-12}$, and the upper bound of the correction term $\Delta \dot{P}_{\mathrm{b}, \mathrm{gal}}=(-0.027+0.005) \times 10^{-12}$, in order to compute the value $\left(\dot{P}_{\mathrm{b}}-\Delta \dot{P}_{\mathrm{b}, \mathrm{gal}}\right)$ to be inserted in the LHS of Eq. (81). We then get

$\tilde{\varepsilon}=8.9 \times 10^{-5}$,

which means $|\varepsilon| \simeq 5.4 \times 10^{-4}$. Recalling now that $M=$ $m_{1}+m_{2}=2.828 \times M_{\odot}$, this translates into the bound

$|\beta| \lesssim 2 \times 10^{71}$,

which is tighter than the bound (76) coming from "standard" Mercury observations, but weaker than the "Messenger bound" of the previous section. However, note that we do not have the caveat of the error bounds on $J_{2}$ here. 
Finally, once again, it can easily be checked that the expansion (69) is fully convergent also when the data of the pulsar PSR B 1913+16 are inserted, as well as that it is convergent in the solar system field.

\section{Comparison with other bounds for $\beta$}

We shall now compare our bounds on $\beta$, obtained in the previous sections, with existing bounds.

Among the papers which make use of a specific representation of the fundamental commutator (see Refs. [21,22,30]), one can find the following bounds:

(a) $\beta<10^{36}$ (from the Lamb shift, Vagenas, PRL 2008), then refined as $\beta<10^{20}$ (Vagenas, PRD 2011);

(b) $\beta<10^{50}$ (from the Landau levels, Vagenas, PRL 2008), then refined in $\beta<10^{46}$ (Vagenas, PRD 2011);

(c) $\beta<10^{34}$ (from the charmonium, Vagenas, PRD 2011);

(d) $\beta<10^{21}$ (for a scanning tunneling microscope, Vagenas, PRL 2008);

(e) $\beta<10^{34}$ (energy difference in hydrogen levels $1 \mathrm{~S}-2 \mathrm{~S}$, Tkachuk, PRA 2010).

(f) In the same category is also the paper of Bonaldi et al. [19], which probes the free evolution of micro and nano mechanical oscillators with masses around the Planck mass, and places a bound of $\beta<10^{18}$ or even lower.

We then have a group of bounds obtained without postulating a specific representation of the fundamental commutator, but rather based directly on the deformed uncertainty relations. The first is coming from the lack of observed deviations from the standard model theory at the electroweak scale $(100 \mathrm{GeV})$. In fact, Eq. (17) can be promptly cast in the form

$\Delta x \Delta p \geq \frac{\hbar}{2}\left[1+\beta\left(\frac{E_{\mathrm{ew}}}{E_{\mathrm{p}}}\right)^{2}\right]$.

The absence of deviations from the standard Heisenberg principle at the electroweak scale then implies $\beta\left(E_{\text {ew }} / E_{\mathrm{p}}\right)^{2} \ll 1$, which means $\beta \ll 10^{34}$.

A different bound that we can find in the same group comes from the gravitational bar detectors (designed to reveal gravitational waves). In Ref. [18], the AURIGA detector is used to set limits on $\beta$. The uncertainty relation (17) together with the standard Hamiltonian of the harmonic oscillator gives the minimal energy of the resonant bar. A measure of the lowest energy level of the first longitudinal mode of AURIGA finally yields $\beta<10^{33}$.

It is important to remark that the above groups of bounds do not explicitly involve the gravitational interaction at any point of their derivation. As we wrote in the Introduction, gravitation is considered in Refs. [24,27], where the authors arrive at bounds on $\beta$ by postulating a deformation of the Poisson brackets which resembles the deformation of the quantum commutator. Leaving aside the model of Chang, which does not reproduce standard GR when $\beta \rightarrow 0$, Ref. [27] reports the bound $\beta<10^{26}$ from the gravitational redshift, $\beta<10^{19}$ from the law of the reciprocal actions, and, most importantly, $\beta<10^{19}$ from the universality of free fall (namely, the absence of violation of the equivalence principle). As we show in Appendix A, however, it should be stressed that this violation is uniquely due to the, somewhat arbitrary, assumption that the classical Poisson brackets should be deformed in the same way as the quantum commutator. The plausibility of such a hypothesis remains at least questionable. $^{2}$

From the above survey, the present paper emerges as the only one which considers explicitly gravitational effects, without postulating a specific (non-linear) representation of the fundamental commutator, and without requiring a violation of the equivalence principle.

\section{Conclusions}

We have shown that a suitable deformation of the Schwarzschild metric can reproduce the Hawking temperature for a black hole, when this is computed from a GUP. We have found in this way an analytic relation between the deformation parameter of the metric $\varepsilon$ and the usual GUP deformation parameter $\beta$. In particular, when $\beta \rightarrow 0$, we correctly recover GR and standard quantum mechanics. Neither the geodesic equation, nor the equivalence principle is violated, for any value of $\beta$ or $\varepsilon$.

Well-known astronomical measurements, in the Solar system as well as in binary pulsar systems, allowed us to put constraints on the parameter $\beta$. This direction seems to point toward promising research: at present we just deformed the Schwarzschild solution, but a future possibility is to deform the full field equations of GR, in order to get, among other things, a more stringent bound on the GUP parameter $\beta$. We would like to conclude by emphasizing once again that, although in the existing literature one can find bounds on $\beta$ much tighter than those obtained in this paper, they seem to depend, at least partially, either on a specific (non-linear) representation of the deformed commutator, or on the hypothesis of a deformation of the Poisson brackets, which implies a

\footnotetext{
${ }^{2}$ In Refs. [56-58], a GUP was straightforwardly derived from a quantum description of the horizon and shown to be incompatible with the semiclassical behavior, as the uncertainty in the size of the horizon of a macroscopically large black hole would be comparable with the horizon size itself.
} 
violation of the equivalence principle. The line of reasoning presented in this paper avoids these possible difficulties.

Open Access This article is distributed under the terms of the Creative Commons Attribution 4.0 International License (http://creativecomm ons.org/licenses/by/4.0/), which permits unrestricted use, distribution, and reproduction in any medium, provided you give appropriate credit to the original author(s) and the source, provide a link to the Creative Commons license, and indicate if changes were made.

Funded by SCOAP ${ }^{3}$.

\section{Appendix A: Modified Poisson brackets and equivalence principle}

We shall show here that a deformation of the classical Newtonian (i.e. non-covariant) Poisson brackets implies a violation of the equivalence principle. In Refs. [27-29] as well as [2426], Poisson brackets are deformed in the same fashion as the quantum commutators derived from a GUP.

Now, considering just a one dimensional system, to keep the calculation simple, we can write the Poisson brackets for one pair of canonical variables as

$\{q, p\}=1+\beta p^{2}, \quad\{q, q\}=\{p, p\}=0$.

It is then easy to show that for any regular function $H(q, p)$, the following hold:

$\{q, H\}=\left(1+\beta p^{2}\right) \frac{\partial H}{\partial p}$,

$\{p, H\}=-\left(1+\beta p^{2}\right) \frac{\partial H}{\partial q}$.

A point-like particle of mass $m$ moving in a Newtonian potential is described by the Hamiltonian (we assume $M \gg m$ )

$H=\frac{p^{2}}{2 m}-\frac{G_{\mathrm{N}} M m}{q}$,

and therefore evolves according to the equations of motion

$\dot{q}=\{q, H\}=\left(1+\beta p^{2}\right) \frac{p}{m}$,

$\dot{p}=\{p, H\}=-\left(1+\beta p^{2}\right) \frac{G_{\mathrm{N}} M m}{q^{2}}$.

From the first equation, we get

$m \dot{q}=p+\beta p^{3}$,

which implies

$m \ddot{q}=\left(1+3 \beta p^{2}\right) \dot{p}$,

and, using now the second of Eq. (90), we have, to first order in $\beta$,

$\ddot{q} \simeq-\left(1+4 \beta p^{2}\right) \frac{G_{\mathrm{N}} M}{q^{2}}$.
Equation (91) can be solved for $p$ to the first order in $\beta$, yielding

$p \simeq m \dot{q}-\frac{\beta(m \dot{q})^{3}}{1+3 \beta(m \dot{q})^{2}}$.

Finally, to first order in $\beta$, we have the equation of motion

$\ddot{q} \simeq-\left(1+4 \beta(m \dot{q})^{2}\right) \frac{G_{N} M}{q^{2}}$.

Clearly, the trajectory of a test particle of mass $m$ will depend on $m$, which signals a violation of the equivalence principle. This violation has nothing to do with GR, or the geodesic equation, or the covariant formalism, but strictly follows from the modified Poisson brackets (87).

\section{Appendix B: Non-relativistic analog}

We display here a non-relativistic analog of the deformation procedure followed in the main text. For a given metric like the one in Eq. (3) we know that, in the situation of a weak, stationary field, and for slowly moving particles, we can define the effective (Newtonian) potential (see, e.g., [43])

$V(r)=\frac{1}{2}[F(r)-1]$,

and the Hamiltonian of a particle of mass $m$, moving in this potential, can be written as

$H=\frac{p^{2}}{2 m}+m V(q)$.

We assume undeformed, standard Poisson brackets

$\{q, p\}=1, \quad\{q, q\}=\{p, p\}=0$,

therefore the equations of motion are the usual ones,

$\dot{q}=\{q, H\}=\frac{\partial H}{\partial p}=\frac{p}{m}$,

$\dot{p}=\{p, H\}=-\frac{\partial H}{\partial q}=-m \frac{\partial V}{\partial q}$,

which yield the equation of motion for $q$

$\ddot{q}=-\frac{\partial V}{\partial q}$.

The above clearly preserves the equivalence principle for any kind of potential $V(q)$. In particular, by choosing a deformed metric as in Eq. (29), we have

$\ddot{q}=-\frac{G_{\mathrm{N}} M}{q^{2}}+\varepsilon \frac{G_{\mathrm{N}}^{2} M^{2}}{q^{3}}$,

which does not depend on $m$ and the equivalence principle is not violated. 


\section{References}

1. H.S. Snyder, Phys. Rev. 71, 38 (1947)

2. C.N. Yang, Phys. Rev. 72, 874 (1947)

3. C.A. Mead, Phys. Rev. B 135, 849 (1964)

4. F. Karolyhazy, Nuovo Cim. A 42, 390 (1966)

5. D. Amati, M. Ciafaloni, G. Veneziano, Phys. Lett. B 197, 81 (1987)

6. D.J. Gross, P.F. Mende, Phys. Lett. B 197, 129 (1987)

7. D. Amati, M. Ciafaloni, G. Veneziano, Phys. Lett. B 216, 41 (1989)

8. K. Konishi, G. Paffuti, P. Provero, Phys. Lett. B 234, 276 (1990)

9. G. Veneziano, in Quantum Gravity Near the Planck Scale. Proceedings of PASCOS'90, Boston, 1990, p. 486 (unpublished)

10. M. Maggiore, Phys. Lett. B 304, 65 (1993)

11. A. Kempf, J. Math. Phys. 35, 4483 (1994)

12. A. Kempf, G. Mangano, R.B. Mann, Phys. Rev. D 52, 1108 (1995)

13. M. Bojowald, A. Kempf, Phys. Rev. D 86, 085017 (2012). arXiv: 1112.0994

14. F. Scardigli, Phys. Lett. B 452, 39 (1999)

15. R.J. Adler, D.I. Santiago, Mod. Phys. Lett. A 14, 1371 (1999)

16. F. Scardigli, R. Casadio, Class. Quantum Gravity 20, 3915 (2003)

17. C. Brukner et al., Nat. Phys. 8, 393 (2012)

18. F. Marin, M. Cerdonio et al., Nat. Phys. 9, 71 (2013)

19. M. Bawaj, C. Biancofiore, F. Marin et al., Nat. Commun. 6, 7503 (2015). arXiv:1411.6410

20. F. Brau, J. Phys. A 32, 7691 (1999)

21. S. Das, E. Vagenas, Phys. Rev. Lett. 101, 221301 (2008)

22. A.F. Ali, S. Das, E. Vagenas, Phys. Rev. D 84, 044013 (2011)

23. P. Pedram, K. Nozari, S.H. Taheri, JHEP 1103, 093 (2011)

24. L.N. Chang, D. Minic, N. Okamura, T. Takeuchi, Phys. Rev. D 66, 026003 (2002)

25. K. Nozari, S. Akhshabi, Chaos Solitons Fractals 37, 324 (2008)

26. P. Pedram, Phys. Lett. B 718, 638 (2012)

27. S. Ghosh, Class. Quantum Gravity 31, 025025 (2014)

28. S. Pramanik, S. Ghosh, Int. J. Mod. Phys. A 28(27), 1350131 (2013)

29. S. Pramanik, Phys. Rev. D 90, 024023 (2014). arXiv: 1404.2567

30. C. Quesne, V.M. Tkachuk, Phys. Rev. A 81, 012106 (2010)

31. M. Walker, J. Math. Phys. (N.Y.) 11, 2280 (1970)

32. A. Zee, Quantum Field Theory in a Nutshell (Princeton University Press, Princeton, 2003)

33. W. Heisenberg, Z. für Phys. 43, 172 (1927)

34. F. Scardigli, Nuovo Cim. B 110, 1029 (1995)

35. R.J. Adler, P. Chen, D.I. Santiago, Gen. Relativ. Gravit. 33, 2101 (2001)

36. M. Cavaglia, S. Das, Class. Quantum Gravity 21, 4511 (2004)
37. M. Cavaglia, S. Das, R. Maartens, Class. Quantum Gravity 20, L205 (2003)

38. L. Susskind, J. Lindesay, An Introduction to Black Holes, Information, and the String Theory Revolution (World Scientific, Singapore, 2005). (See chapter 10)

39. K. Nouicer, Class. Quantum Gravity 24, 5917 (2007)

40. F. Scardigli, Glimpses on the micro black hole Planck phase. arXiv:0809.1832

41. G. Dvali, C. Gomez, Black hole's quantum N-portrait. arXiv: 1112.3359

42. P. Jizba, H. Kleinert, F. Scardigli, Phys. Rev. D 81, 084030 (2010)

43. S. Weinberg, Gravitation and Cosmology (Wiley, New York, 1972)

44. J.M. Gerard, S. Pireaux, The observable light deflection angle. arXiv:gr-qc/9907034

45. C.M. Will, Living Rev. Relativ. 9, 3 (2006). arXiv:1403.7377v1. http://www.livingreviews.org/lrr-2006-3. Accessed 10 Sept 2015

46. S.S. Shapiro, J.L. Davis, D.E. Lebach, J.S. Gregory, Phys. Rev. Lett. 92, 121101 (2004)

47. S.B. Lambert, C. Le Poncin-Lafitte, Astron. Astrophys. 499, 331 (2009). arXiv:0903.1615

48. http://en.wikipedia.org/wiki/Hipparcos. http://sci.esa.int/gaia/. Accessed 10 Sept 2015

49. See the paper of Johann Georg von Soldner (1801) translated and reproduced in S. Jaki, Found. Phys. 8(11-12), 927 (1978)

50. I.I. Shapiro, in Solar System Tests of General Relativity: Recent Results and Present Plans, eds. by N. Ashby, D.F. Bartlett, W. Wys. General Relativity and Gravitation. Proceedings of the 12th International Conference on General Relativity and Gravitation, University of Colorado at Boulder, 28 July 1989 (Cambridge University Press, Cambridge, 1990), pp. 313-330

51. A.K. Verma, A. Fienga, J. Laskar, H. Manche, M. Gastineau, Astron. Astrophys. 561, A115 (2014)

52. J.M. Weisberg, D.J. Nice, J.H. Taylor, Astrophys. J. 722, 1030 (2010)

53. T. Damour, N. Deruelle, Ann. Inst. Henri Poincare (Phys. Theor.) 44, 263 (1986)

54. J.H. Taylor, J.M. Weisberg, Astrophys. J. 345, 434 (1989)

55. T. Damour, J.H. Taylor, Astrophys. J. 366, 501 (1991)

56. R. Casadio, F. Scardigli, Eur. Phys. J. C 74, 2685 (2014)

57. R. Casadio, Horizons and non-local time evolution of quantum mechanical systems. arXiv: 1411.5848

58. R. Casadio, A. Giugno, O. Micu, A. Orlandi, Phys. Rev. D 90(8), 084040 (2014) 